\title{
Simple Observer Based Synchronization of Lorenz System with Parametric Uncertainty
}

\author{
Manish Kumar Vaishnav ${ }^{1}$, Dr. B.B. Sharma ${ }^{2}$ \\ ${ }^{1,2}$ (EED, National Institute of Technology, Hamirpur (H.P), India)
}

\begin{abstract}
In this paper, observer based synchronization of chaotic Lorenz system is presented. The virtual system concept is used for synchronization of observer states to actual system states. First, synchronization is presented without any uncertainty in system parameters and further synchronization is achieved for the case with uncertainty in system parameters. Adaptation law for uncertain parameters are developed as a consequence of stability analysis. Convergences of uncertain parameters are also analyzed. Simulation results are presented in the end to verify the synchronization of observer states to actual system states.
\end{abstract}

Keywords: Contraction theory, Exponential convergence, Observer, Parametric uncertainty, Synchronization, Virtual system.

\section{Introduction}

Since last few decades, synchronization \& stability of chaotic system have been explored by many researchers [1-4]. Synchronization of two chaotic system means that system trajectories of both the system are varying in synchronized way keeping in view the significant applications of chaotic systems in different fields, many chaotic systems are studied and analyzed. In this paper, observer based synchronization is presented for chaotic Lorenz system. Chaotic Lorenz system is described by set of differential equations proposed by meteorologist/mathematician Edward N. Lorenz, who was studying thermal variations in an air cell underneath a thunderhead. Since its inception, the Lorenz system has become one of the most widely studied system of ODEs because of its wide range of behaviour.

In the present paper contraction theory results are utilized to address synchronization of Lorenz system. Contraction theory is recently developed tool for analyzing the stability and synchronization of nonlinear control system. Some of important results of contraction theory can be found in literature [5-10] \& references therein. In observer based synchronization approach, the actual system states are reconstructed by utilizing the information available at the actual system output. The output may be function of one or more state or the combination of states. More information related to such approaches can be found in literature [11-14]. In this paper, it is assumed that the output is the function of only one state of actual system. In most of the cases Lyapunov based analysis is used for observer based synchronization of chaotic system. Here, Contraction theory is used for the synchronization of the Lorenz system. The advantage of this method is that, there is no issue of selection of energy function and it handles the synchronization problem effectively. Adaptation laws for uncertain parameter are developed in terms of estimated states. Here adaptation laws are obtained while establishing stability using virtual system concept.

This paper is outlined as follows: In section-2, preliminaries of contraction theory and virtual system are presented. In section-3, problems are formulated for synchronization of chaotic Lorenz system for both parametric certainty and parametric uncertainty cases. In section-4, simulation results are presented to verify the synchronization of observer states to actual system states. Finally, conclusion is presented in section-5 of paper.

Consider a nonlinear system

\section{Preliminaries}

$\dot{\mathbf{x}}=\mathbf{f}(\mathbf{x}, t)(1)$

Where $\mathbf{x} \in \mathfrak{R}^{m}$ and $\mathbf{f}(\mathbf{x}, t)$ is continuous differentiable function such that, $\mathbf{f}:(m \times 1)$ vector function. The main definition and theorem of contraction are taken from [15] and reproduced here for the clarity purpose. Let $\delta \mathbf{x}$ be the virtual displacement in $\mathbf{X}$, first variation in $\mathbf{X}$ will be

$\delta \dot{\mathbf{x}}=\frac{\partial \mathbf{f}(\mathbf{x}, t)}{\partial \mathbf{x}} \delta \mathbf{x} \quad$ (2) Now taking the time derivative of squared distance

$\frac{d}{d t}\left(\delta \mathbf{x}^{T} \delta \mathbf{x}\right)=2 \delta \mathbf{x}^{T} \delta \dot{\mathbf{x}}=2 \delta \mathbf{x}^{T} \frac{\partial \mathbf{f}}{\partial \mathbf{x}} \delta \mathbf{x} \leq 2 \lambda_{m}(\mathbf{x}, t) \delta \mathbf{x}^{T} \delta \mathbf{x}(3)$ 
Where $\frac{\partial \mathbf{f}}{\partial \mathbf{x}}$ represents the Jacobian matrix $\mathbf{J}$ of the system (1) and $\lambda_{m}(\mathbf{x}, t)$ represents the largest eigenvalue of the symmetric part of Jacobian matrix $\mathbf{J}$. If $\lambda_{m}(\mathbf{x}, t)$ is strictly uniformly negative definite (UND), then any infinitesimal length converges exponentially to zero. Then all trajectories of the system will converge together exponentially.

Definition 1. A region of the state space is called a contraction region with respect to a uniformly positive definite metric $\mathbf{M}(\mathbf{x}, t)=\Theta^{T}(\mathbf{x}, t) \Theta(\mathbf{x}, t)$ where $\Theta$ stands for a differential coordinate transformation matrix, if equivalently

$\mathbf{F}=\left(\dot{\Theta}+\Theta \frac{\partial \mathbf{f}}{\partial \mathbf{x}}\right) \Theta^{-1}(4)$

Is uniformly negative definite (UND). This leads to the following convergence result.

Lemma 1.For system (1), any trajectory which starts in a ball of constant radius centered about a given trajectory and contained at all the time in a contraction region, remains in that ball and converges exponentially to given trajectory. Global exponential convergence to given trajectory is guaranteed if whole state space region is contracting.

The following Lemma is used to analyze the convergence of observer states to actual system states and taken from [16].

Lemma 2.If a virtual system defined for actual system and observer system along with parameter adaptationlaw, then incremental behaviour in terms of virtual displacement could be represented in matrix form as

$$
\left[\begin{array}{l}
\delta \dot{\boldsymbol{\Phi}} \\
\ldots \\
\delta \dot{\boldsymbol{\theta}}
\end{array}\right]=\left[\begin{array}{ccc}
\mathbf{J}_{11} & : & \mathbf{Q}(\hat{\mathbf{y}}, t) \\
\ldots & \ldots & \ldots \\
-\mathbf{Q}^{T}(\hat{\mathbf{y}}, t) & : & \mathbf{0}
\end{array}\right]\left[\begin{array}{c}
\delta \boldsymbol{\Phi} \\
\ldots \\
\delta \boldsymbol{\theta}
\end{array}\right](5)
$$

where $\mathbf{Q}(\hat{\mathbf{y}}, t)$ is a nonlinear regression matrix function of observer output $\hat{\mathbf{y}}, \boldsymbol{\Phi}$ and $\boldsymbol{\theta}$ represents state vector and uncertain parameter vector of virtual system, respectively, then system in (5) would be semi contracting in nature subject to following conditions:

(i) Jacobian of virtual system dynamics w.r.t. its state $\boldsymbol{\Phi}$ i.e. submatrix $\mathbf{J}_{\mathbf{1 1}}$ is uniformly negative definite (UND).

(ii) All the variables involved in (5) are bounded.

Semi-contracting nature of virtual system implies that particular solutions of the virtual system converges to each other asymptotically with parameter estimates remaining bounded i.e. $\hat{\mathbf{x}} \rightarrow \mathbf{X}$ as time $t \rightarrow \infty$ and estimate vector $\hat{\theta}$ remains bounded.

II.1 Virtual System Theory

Let the dynamics of a nonlinear system is given as $\dot{\mathbf{x}}=-\eta(\mathbf{x}, \boldsymbol{\theta}, t) \mathbf{x}$

Where $\mathbf{x} \in \mathfrak{R}^{m}, \boldsymbol{\theta}$ is parameter of the system and function $\eta(\mathbf{x}, \boldsymbol{\theta}, t) \geq \alpha \mathbf{I}>0$.

Now virtual system is defined as

$\dot{\mathbf{y}}=-\eta(\mathbf{x}, \boldsymbol{\theta}, t) \mathbf{y}(7)$

In differential frame work

$$
\delta \dot{\mathbf{y}}=-\eta(\mathbf{x}, \boldsymbol{\theta}, t) \delta \mathbf{y}
$$

One can see that the virtual system is contracting w.r.t. $\mathbf{y}$ using the basic results of contraction. Since actual system is a particular solution of the virtual system, so the actual system states will also converge together, exponentially.

In case of observer design, virtual system is defined such as both actual system and observer are particular solutions of the virtual system. For actual system as

$\dot{\mathbf{x}}=\mathbf{x}+u_{(8)}$

Let observer be defined as 
$\dot{\hat{\mathbf{x}}}=\hat{\mathbf{x}}+u+\mathbf{L}(\mathbf{x}-\hat{\mathbf{x}})$

Where $\mathrm{u}$ is control input and $\mathbf{L}$ is gain of the observer, selected as $\mathbf{L}>1$.

Let the virtual system is defined as

$\dot{\mathbf{z}}=\mathbf{z}+u+\mathbf{L}(\mathbf{x}-\mathbf{z})(10)$

The value of $\mathbf{L}$ is selected such that the Jacobian of virtual system is uniformly negative definite (UND). It will establish contracting nature of virtual system and further it will lead to exponential convergence of observer states to actual system states.

III. The Investigation Of Chaotic Lorenz System's Synchronization Based On Contraction Theory

The equations of Lorenz system is described as follows:

$\left.\begin{array}{l}\dot{x}_{1}=-a x_{1}+a x_{2} \\ \dot{x}_{2}=r x_{1}-x_{1} x_{3}-x_{2} \\ \dot{x}_{3}=x_{1} x_{2}-b x_{3}\end{array}\right\}(11)$

Where $a, \mathrm{r}$ and $\mathrm{b}$ are real constant parameters and $x_{1}, x_{2}$ and $x_{3}$ are variables.

Let the measurable output of Lorenz's system be given as

$y=\mathbf{c x}=\left[\begin{array}{lll}1 & 0 & 0\end{array}\right] \mathbf{x} ;(12)$

The system is chaotic when $a=10, \mathrm{r}=30$ and $b=8 / 3$. Initial conditions for the system are taken as $\left[\begin{array}{lll}-3 & -7 & 5\end{array}\right]^{T}$ and simulation is run for 80 seconds with step size of 0.01 .The behaviour of the system is shown in Fig.(1). Three dimensional phase portrait is shown in Fig.1 (a). The open loop response of the system to initial condition is shown in Figs.1 (b)-(d).

(a)

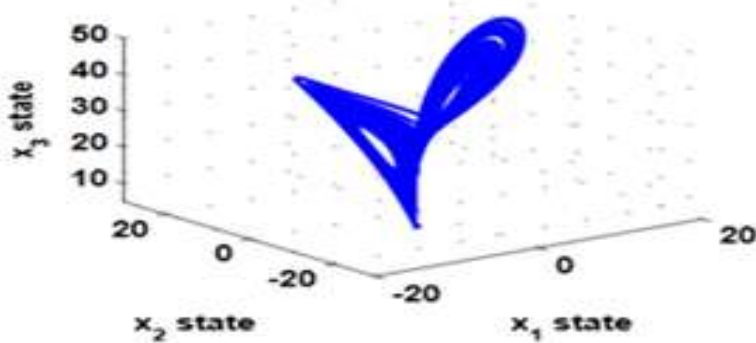

(c)

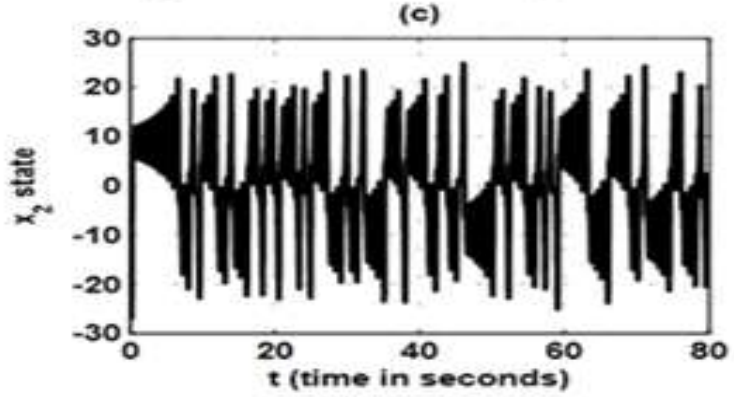

(b)

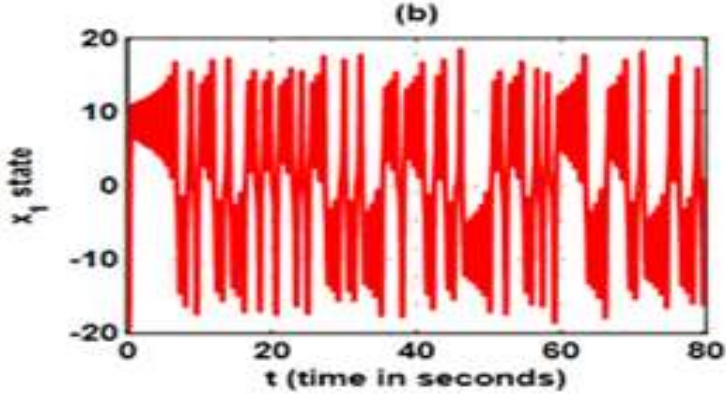

(d)

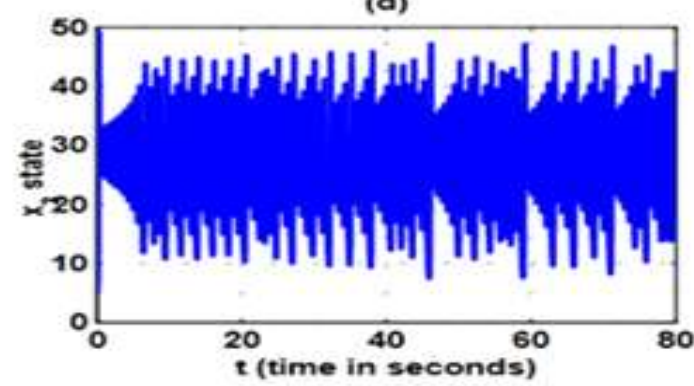

Fig.1 Lorenz's system without controller: (a) Phase portrait in three dimensions and (b) - (d) time variation of state trajectories

III.1 Observer design for chaotic Lorenz system for without parametric uncertainty

The structure of the observer for the system (11) can be taken as 


$$
\left.\begin{array}{l}
\dot{\hat{x}}_{1}=-a \hat{x}_{1}+a \hat{x}_{2}+L_{1}(y-\hat{y}) \\
\dot{\hat{x}}_{2}=r \hat{x}_{1}-\hat{x}_{2}-\hat{x}_{1} \hat{x}_{3}+L_{2}(y-\hat{y}) \\
\dot{\hat{x}}_{3}=\hat{x}_{1} \hat{x}_{2}-b \hat{x}_{3}+L_{3}(y-\hat{y})
\end{array}\right\}
$$

And the output of the observer is given by $\hat{y}=\mathbf{C} \hat{\mathbf{x}}=\left[\begin{array}{lll}1 & 0 & 0\end{array}\right] \hat{\mathbf{x}}(14)$

Where $L_{1}, L_{2}$ and $L_{3}$ represents the gain of full order observer. Select gains of observer such that observer states converge exponentially to actual system states. Let the virtual system for actual system (11) and observer system (13) be selected as

$$
\left.\begin{array}{l}
\dot{\Phi}_{1}=-a \Phi_{1}+a \Phi_{2}+L_{1}\left(y-y_{\Phi}\right) \\
\dot{\Phi}_{2}=r \Phi_{1}-\Phi_{2}-\Phi_{1} \Phi_{3}+L_{2}\left(y-y_{\Phi}\right) \\
\dot{\Phi}_{3}=\Phi_{1} \Phi_{2}-b \Phi_{3}+L_{3}\left(y-y_{\Phi}\right)
\end{array}\right\}
$$

And the output of the virtual system is given by

$$
y_{\Phi}=\mathbf{C} \Phi=\left[\begin{array}{lll}
1 & 0 & 0
\end{array}\right] \Phi
$$

Now using the definition of $y$ and $y_{\Phi}$ in virtual system (15) and defining the virtual displacement $\delta \Phi$ as

$$
\delta \dot{\Phi}=\mathbf{J} \delta \Phi(17)
$$

Where $\mathbf{J}$ represents the Jacobian matrix of virtual system and given as

$$
\mathbf{J}=\left[\begin{array}{ccc}
-a-L_{1} & a & 0 \\
r-\Phi_{3}-L_{2} & -1 & -\Phi_{1} \\
\Phi_{2}-L_{3} & \Phi_{1} & -b
\end{array}\right]
$$

The symmetric part of Jacobian $\mathbf{J}_{\mathrm{s}}=\frac{1}{2}\left(\mathbf{J}+\mathbf{J}^{T}\right)$ can be represented as

$$
\mathbf{J}_{s}=\left[\begin{array}{ccc}
-a-L_{1} & \frac{\left(a+r-\Phi_{3}-L_{2}\right)}{2} & \frac{\left(\Phi_{2}-L_{3}\right)}{2} \\
\frac{\left(a+r-\Phi_{3}-L_{2}\right)}{2} & -1 & 0 \\
\frac{\left(\Phi_{2}-L_{3}\right)}{2} & 0 & -b
\end{array}\right]
$$

The gains $L_{i} ; i=1,2,3$ are selected suitably that ensure $\mathbf{J}_{s}$ to be UND in nature, so that contracting nature of virtual system is established. Since actual system and observer are particular solution of virtual system, so these will converge to each other, exponentially. To ensure UND nature of $\mathbf{J}_{s}$ following condition should be satisfied.

(i) $L_{1}>-a$

(ii) $\left(a+L_{1}\right)>\frac{\left(a+r-\Phi_{3}-L_{2}\right)^{2}}{4}$

(iii) $\frac{\left(\Phi_{2}-L_{3}\right)^{2}}{4}<\left(a+L_{1}\right) b-\frac{\left(a+r-\Phi_{3}-L_{2}\right)^{2}}{4} b$ 

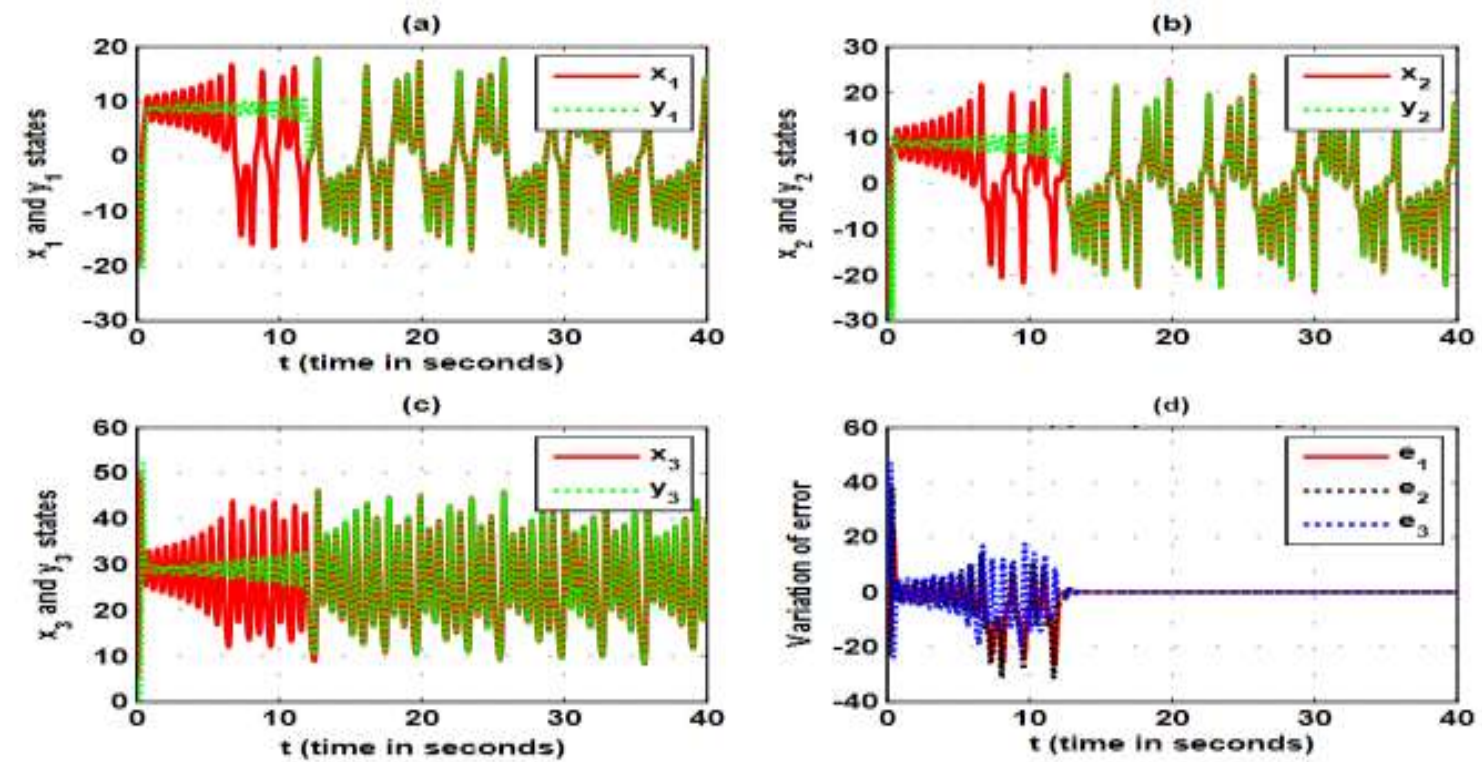

Fig. 2 Convergence of behaviour for Lorenz's systemwith certain parameters: (a)-(c) time variation of state trajectories and (d) variation of state estimation error.

\section{III.2 Observer design for chaotic Lorenz system for parametric uncertainty}

Here, it is assumed that parameter ' $a$ ' of Lorenz system is uncertain and it is assumed that uncertain parameter appears in state equation corresponding to measurable variable in the state dynamics. So modified observer dynamics for uncertain parameter case is presented as

$$
\left.\begin{array}{l}
\dot{\hat{x}}_{1}=-\hat{a} \hat{x}_{1}+\hat{a} \hat{x}_{2}+L_{1}(y-\hat{y}) \\
\dot{\hat{x}}_{2}=r \hat{x}_{1}-\hat{x}_{2}-\hat{x}_{1} \hat{x}_{3}+L_{2}(y-\hat{y}) \\
\dot{\hat{x}}_{3}=\hat{x}_{1} \hat{x}_{2}-b \hat{x}_{3}+L_{3}(y-\hat{y})
\end{array}\right\}
$$

Where $\hat{a}$ represents the estimated value of parameter $a$ and parametric error is given by $\tilde{a}=\hat{a}-a$.Using this definition, the observer dynamics is modified as

$$
\left.\begin{array}{l}
\dot{\hat{x}}_{1}=-a \hat{x}_{1}+a \hat{x}_{2}-\tilde{a} \hat{x}_{1}+\tilde{a} \hat{x}_{2}+L_{1}(y-\hat{y}) \\
\dot{\hat{x}}_{2}=r \hat{x}_{1}-\hat{x}_{2}-\hat{x}_{1} \hat{x}_{3}+L_{2}(y-\hat{y}) \\
\dot{\hat{x}}_{3}=\hat{x}_{1} \hat{x}_{2}-b \hat{x}_{3}+L_{3}(y-\hat{y})
\end{array}\right\}
$$

Let the adaptation law for uncertain parameter be

$$
\dot{\hat{a}}=\left(-\hat{x}_{1}+\hat{x}_{2}\right)\left(x_{1}-\hat{x}_{1}\right)
$$

Defining a new virtual system as

$$
\left.\begin{array}{l}
\dot{\Phi}_{1}=-a \Phi_{1}+a \Phi_{2}-\tilde{a}_{1} \hat{x}_{1}+\tilde{a}_{1} \hat{x}_{2}+L_{1}\left(y-y_{\Phi}\right) \\
\dot{\Phi}_{2}=r \Phi_{1}-\Phi_{2}-\Phi_{1} \Phi_{3}+L_{2}\left(y-y_{\Phi}\right) \\
\dot{\Phi}_{3}=\Phi_{1} \Phi_{2}-b \Phi_{3}+L_{3}\left(y-y_{\Phi}\right)
\end{array}\right\}
$$


And the adaptation law as

$\dot{\bar{a}}=\left(-\hat{x}_{1}+\hat{x}_{2}\right)\left(x_{1}-\Phi_{1}\right)$

Where $\bar{a}$ is the parameter of virtual system and parametric error is defined as $\tilde{a}_{1}=\bar{a}-a$. The Jacobian matrix in this case will be

$\mathbf{J}=\left[\begin{array}{cccc}-a-L_{1} & a & 0 & \left(-\hat{x}_{1}+\hat{x}_{2}\right) \\ r-\Phi_{3}-L_{2} & -1 & -\Phi_{1} & 0 \\ \Phi_{2}-L_{3} & \Phi_{1} & -b & 0 \\ -\left(-\hat{x}_{1}+\hat{x}_{2}\right) & 0 & 0 & 0\end{array}\right]$

The symmetric part of Jacobian $\mathbf{J}_{\mathbf{s}}=\frac{1}{2}\left(\mathbf{J}+\mathbf{J}^{T}\right)$ can be represented as

$\mathbf{J}_{s}=\left[\begin{array}{cccc}-a-L_{1} & \frac{\left(a+r-\Phi_{3}-L_{2}\right)}{2} & \frac{\left(\Phi_{2}-L_{3}\right)}{2} & 0 \\ \frac{\left(a+r-\Phi_{3}-L_{2}\right)}{2} & -1 & 0 & 0 \\ \frac{\left(\Phi_{2}-L_{3}\right)}{2} & 0 & -b & 0 \\ 0 & 0 & 0 & 0\end{array}\right]$

By selecting the suitable values of gain $L_{i} ; i=1,2,3$, UND nature of $\mathbf{J}_{\mathbf{1 1}}$ system can be ensure. So by using Lemma 2, it can be said that observer states will converge to actual system states. It is not said confidently about the convergence of estimate of uncertain parameter to its actual value. Although uncertain parameter is bounded in our case.

\section{Simulations}

To verify the synchronization of the observer states to actual system states for the case without parametric uncertainty, initial condition for the proposed observer is taken as $\left[\begin{array}{lll}-0.3 & -0.5 & 0.4\end{array}\right]^{T}$.The value of gains $L_{i} ; i=1$, 2, 3, are chosen so that $\mathbf{J}_{s}$ is $\mathrm{UND}$ in nature. The desired values of observer gains are $L_{1}=240, L_{2}=12$ and $L_{3}=8$. Simulation is run for 40 seconds with step size of 0.01 . Initially observer gains are kept zero and switched on at $\mathrm{t}=12$ seconds. The convergence of observer state $\hat{\mathbf{x}}$ to actual state $\mathbf{X}$ is shown in Fig. (2).

In parametric uncertainty case, the initial condition for observer are taken as $\left[\begin{array}{lll}-0.6 & -0.2 & 0.8\end{array}\right]^{T}$. The simulation is run for 40 seconds with step size of 0.01 seconds. Initially, parametric estimate for uncertain parameter ' $a$ ' is taken as zero. Here, observer gains are switched on at $\mathrm{t}=5$ seconds. The convergence behaviour is shown in Fig. (3). The time variation of state trajectories of observer and actual Lorenz's system is presented in Fig. (a)-(c), variation of state estimation error and convergence behaviour of estimates of uncertain parameter are presented in Fig. (d) \& (e), respectively. 
(a)
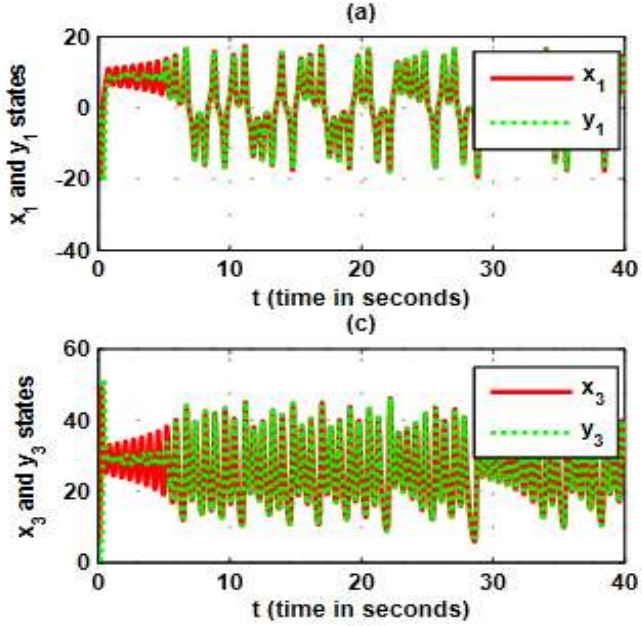

(e)

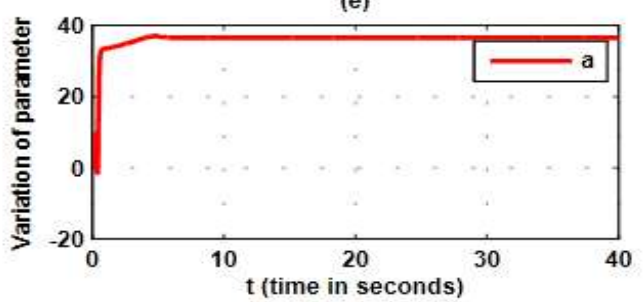

(b)

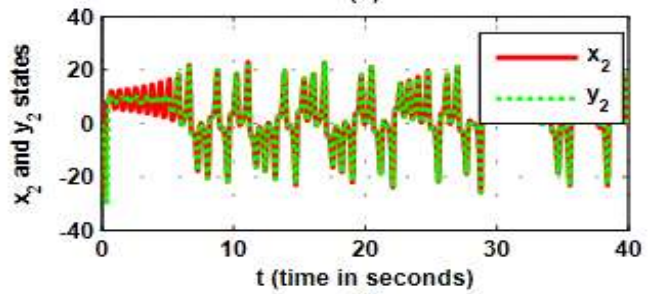

(d)

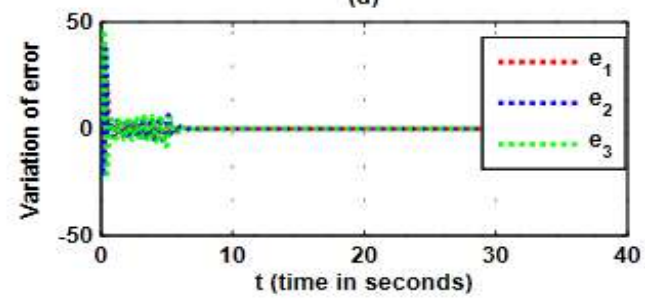

Fig. 3 Convergence behaviour of observer for Lorenz's system with uncertain parameters: (a)-(c) time variation of state trajectories of observer and actual Lorenz's system, (d) variation of state estimation error and (e) convergence behaviour of estimates of uncertain parameter.

\section{Conclusion}

Synchronization scheme for chaotic Lorenz system is presented using observer based methodology. The approach utilizes virtual system theory concept. Contraction theory provides very effective way of establishing synchronization. Parameter adaptation law is derived in terms of estimated states. Finally, establishing simulation results are presented to illustrate the asymptotic converge of observer states to the actual system states. Parametric estimates remains bounded while establishing these results.

\section{References}

[1] Jayaram A., Tadi M.: Synchronization of chaotic systems based on SDRE method. Chaos Solitons Fractals vol.28, Nr.3, pp.707715 (2006).

[2] Carroll T.L. and Pecora L.M.: Synchronizing chaotic circuits. IEEE Trans. vol.38, pp.453-456 (1991).

[3] CarrollT.L. and Pecora L.M.: Cascading synchronized chaotic systems. Physica D 67, 126-140 (1993).

[4] Carroll T.L. and Pecora L.M.: Synchronizing non-autonomous chaotic circuits. IEEE Trans. Circuits Syst. 40, 646 (1995).

[5] Lohmiller W., and Slotine J.J.E.: Control system design for mechanical systems using Contraction theory.IEEE Trans. Automatic Control, vol.45, No.5 (2000).

[6] Jouffroy J. and Slotine J.J.E.: Methodological remarks on contraction theory. IEEE Conference on Decision and Control Atlantis, Paradise Island, Bahamas, pp. 2537- 2543 (2004).

[7] Jouffroy J., Some ancestors of contraction analysis, Proc. of IEEE Conference on Decision and Control, pp.5450-5455, Seville, Spain (2005).

[8] B. B. Sharma and I. N. Kar: Design of asymptotically convergent frequency estimator using contraction theory. IEEE Trans. on Automatic Control, vol.53, No 8, pp.1932-1937 (2008)

[9] B.B.Sharma and I.N.Kar: Contraction theory based recursive design of stabilizing controller for a class of nonlinear systems. IET control theory \& applications, pp.1005-1018 (2010).

[10] B.B.Sharma and I. N.Kar: Contraction based adaptive control of a class of nonlinear systems. Proc. American control conference, pp. 808-813 (2009).

[11] Liao T.L., Huang N.S.: An observer based approach for chaotic synchronization and secure communication. IEEE Trans. Circuits Syst.I 46(9), 1144-1149 (1999).

[12] Nijmeijer H., MareelI.M.: An observer looks at synchronization. IEEE Trans. Circuits Syst.I 44(10), 882-890 (1997).

[13] Boutayeb M., Darouach M., Rafaralahy H.: Generalized state-space observers for chaotic synchronization with applications to secure communication. IEEE Trans. Circuits Syst.I 49(3), 345-349 (2002).

[14] Cherrier E., Boutayeb M., Ragot J.: Observers-based synchronization and input recovery for a class of nonlinear chaotic models. IEEE Trans. Circuits Syst.I 53(9), 1977-1986 (2006).

[15] LohmillerW. and SlotineJ.J.E.: On contraction analysis for nonlinear systems. Automatica, vol.34, No.6, pp. 683-696 (1998).

[16] B.B.Sharma and I. N.kar: Observer-based synchronization scheme for a class of chaotic systems using contraction theory.Nonlinear Dynamics, vol.63, No.3, pp. 429-445 (2011). 